\title{
Safingol Hydrochloride
}

National Cancer Institute

\section{Source}

National Cancer Institute. Safingol Hydrochloride. NCI Thesaurus. Code C80327.

The hydrochloride salt of a saturated derivative of sphingosine. As an inhibitor of protein kinase $C(P K C)$, safing ol competitively binds to the regulatory phorbol-binding domain of PKC, a kinase involved in tumorigenesis. This agent has been shown to act synergistically with other chemotherapeutic agents and may potentiate chemotherapy drug-induced apoptosis in vitro and in vivo. 